\title{
POSITIVE SOLUTIONS FOR EVEN-ORDER MULTI-POINT BOUNDARY VALUE PROBLEMS ON TIME SCALES
}

\author{
MUSTAFA GÜNENDI* AND İSMAİL YASLAN** \\ Department of Mathematics, Pamukkale University \\ 20070 Denizli, Turkey \\ E-mail: *mustafa_87875@hotmail.com, ${ }^{* *}$ iyaslan@pau.edu.tr
}

Abstract. In this paper, we consider the nonlinear even-order $m$-point boundary value problems on time scales. We establish the criteria for the existence of at least one and three positive solutions for higher order nonlinear $m$-point boundary value problems on time scales by using Krasnosel'skii's fixed point theorem and Leggett-Williams' fixed point theorem, respectively.

Key Words and Phrases: Boundary value problems, cone, fixed point theorems, positive solutions, time scales.

2010 Mathematics Subject Classification: 34B18, 34N05, 39A10, 47H10.

\section{REFERENCES}

[1] M. Bohner, A. Peterson, Dynamic Equations on Time Scales: An Introduction with Applications, Birkhäuser, Boston, 2001.

[2] M. Bohner, A. Peterson (eds.), Advances in Dynamic Equations on Time Scales, Birkhäuser, Boston, 2003.

[3] A. Dogan, Existence of multiple positive solutions for p-Laplacian multipoint boundary value problems on time scales, Adv. Differ. Eq., 2013(2013), no. 238, 1-23.

[4] D. Guo, V. Lakshmikantham, Nonlinear Problems in Abstract Cones, Academic Press, San Diego, 1988

[5] S. Hilger, Analysis on measure chains - A unified approach to continuous and discrete calculus, Results Math., 18(1990), no. 1-2, 18-56.

[6] V.A. Il'in, E.I. Moiseev, Nonlocal boundary value problem of the first kind for a Sturm Liouville operator in its differential and finite difference aspects, Differ. Eq., 23(1987), no. 7, 803-810.

[7] J. Jiang, L. Liu, Positive solutions for nonlinear second-order m-point boundary-value problems, Electron. J. Differ. Eq., 2009(2009), no. 110, 1-12.

[8] I.Y. Karaca, F. Tokmak, Eigenvalues for iterative systems of nonlinear m-point boundary value problems on time scales, Bound. Value Probl., 2014(2014), no. 63, 1-17.

[9] Y. Liu, W. Zhang, Positive solution for second order multi-point boundary value problem at resonance, Fixed Point Theory, 14(2013), no. 2, 401-411.

[10] R. Luca, On a higher-order m-point boundary value problem, Fixed Point Theory, 13(2012), no. $1,137-145$.

[11] R. Luca, Positive solutions for a higher-order m-point boundary value problem, Mediterr. J. Math., 9(2012), no. 2, 379-392.

[12] R.W. Leggett, L.R. Williams, Multiple positive fixed points of nonlinear operators on ordered Banach space, Indiana Univ. Math. J., 28(1979), no. 4, 673-688. 
[13] R. Ma, Multiple positive solutions for nonlinear m-point boundary value problems, Appl. Math. Comput., 148(2004), no. 1, 249-262.

[14] J. Adem, M. Moshinsky, Self-adjointness of a certain type of vectorial boundary value problems, Bol. Soc. Mat. Mexicana, 7(1950), 1-17.

[15] S. Timoshenko, Theory of Elastic Stability, McGraw-Hill, New York, 1961.

[16] F. Tokmak, I.Y. Karaca, Existence of symmetric positive solutions for a multipoint boundary value problem with sign-changing nonlinearity on time scales, Bound. Value Probl., 2013(2013), no. $52,1-12$.

[17] Y. Wang, Y. Tang, M. Zhao, Multiple positive solutions for a nonlinear 2n-th order m-point boundary value problems, Electron. J. Qualitative Th. Differ. Eq., 2010(2010), no. 39, 1-13.

[18] J. Wang, F. Xu, Nontrivial solutions for nonlinear higher order multi-point boundary value problem on time scales with all derivatives, Appl. Math. Sci., 3(2009), no. 45-48, 2349-2358.

[19] İ. Yaslan, Higher order m-point boundary value problem on time scales, Comput. Math. Appl., 63(2012), no. 3, 739-750.

[20] İ. Yaslan, Existence of positive solutions for even-order m-point boundary value problems on time scales, Electron. J. Differ. Eq., 2013(2013), no. 45, 1-12.

Received: July 24, 2014; Accepted: April 30, 2015. 